\title{
Updated Management of Atrial Fibrillation
}

\author{
Mehmet Ergin ${ }^{1}$, Zerrin Defne Dündar ${ }^{2}$, Semra Aslay ${ }^{1}$, Mücahit Emet ${ }^{3}$ \\ 'Department of Emergency, Near East University Faculty of Medicine, Nicosia, Turkish Republic of Northern Cyprus \\ 2Department of Emergency, Necmettin Erbakan University Meram Faculty of Medicine, Konya, Turkey \\ ${ }^{3}$ Department of Emergency, Atatürk University Faculty of Medicine, Erzurum, Turkey
}

\begin{abstract}
Atrial fibrillation (AF) is the most common sustained cardiac arrhythmia. AF is also associated with increased mortality, stroke and other thromboembolic disorders, cardiac failure and hospital admission, diminished quality of life, exercise intolerance, and left ventricular dysfunction. In the literature, there are many reports about the updated management of AF. This report aims at evaluating the previous guidelines and summarizing the prominent points.

(Eurasian J Emerg Med 2015; 14: 138-41)
\end{abstract}

Keywords: Atrial fibrillation, emergency departments, guideline

\section{Introduction}

Atrial fibrillation (AF) is the most common cardiac arrhythmia, and it is observed in 1-2\% of the general population in Europe. Over six million people live with AF in Europe; however, the actual prevalence is unclear because of asymptomatic AF. Importance of AF is not related with the frequency only. AF becomes prominent with increased death rate, stroke and other thromboembolic events, heart failure and association with the hospitalization, as well as reduction in the quality of life and exercise tolerance, and left ventricle dysfunction $(1,2)$.

It is possible to find many reports about the current approach to manage AF in the literature. There are over 1700 articles in a simple Pubmed search under the title of "AF guideline" (3). An emergency medicine physician may not see the forest for trees in such details. Among these articles, the guidelines of the Canadian Cardiovascular Society (CCS), European Society of Cardiology (ESC), and American College of Cardiology/American Heart Association (ACC/AHA) were prominent $(1,2,4-8)$. However, the presence of a large volume of articles may cause confusion because of the inconsistency between the suggestions. Herein, the need to establish a superior commission by assembling the physicians who are interested in AF management and to review the suggestions is certain. However, different suggestions because of the compliance of the treatment, different healthcare types, and changing patient characteristics are accepted.

The present article summarizes the information included in these guidelines based on the latest guidelines of the three associations.

\section{Definition of AF}

According to the definition of AF in the guideline of the European Society of Cardiology, AF is described by the presence of an irregular RR interval, which is exactly observed on the ECG, and the absence of an exactly differentiated $P$ wave as well as a variable but also a shorter atrial cycle length less than $200 \mathrm{~ms}$ (4-7).

\section{Classification of AF}

A new topic included in the current guidelines is the new classification of AF. All patients in whom AF is diagnosed first are defined as "first diagnosed AF," independently from arrhythmia period and existence and severity of AF-associated symptoms. Paroxysmal AF spontaneously ends within $48 \mathrm{~h}$ in general; however, it may prolong up to 7 days. Forty-eight hours is a significant duration for AF. After this period, the possibility to return to sinus rhythm spontaneously

Correspondence to: Mehmet Ergin; e-mail: drmehmetergin@gmail.com

Received: 17.11.2013 Accepted: 02.04.2015

(c) Copyright 2015 by Emergency Physicians Association of Turkey - Available online at www.eajem.com 
decreased, and anticoagulation treatment should be assessed. Persistent AF is defined when AF attack persists longer than 7 days or should be terminated by chemical or electrical cardioversion. Long standing persistent AF is defined when rhythm control strategy was decided. Permanent AF is the type of AF where both the patient and physician accepted arrhythmia, and the idea of rhythm control strategies disappeared. This classification is useful for clinical management $(1,2)$.

Along with the present classification, you may see definitions of "silent $A F$, incident $A F$, lone $A F$, valvular $A F$, rheumatic $A F$ " for clinical status and etiology (1, 2, 4-8).

Normal progress of AF starts with short and rare attacks and proceeds with longer and frequent attacks. Many patients develop one AF type continuously in time. Only a small group of patients who do not have predisposing factors continue to present with paroxysmal AF despite years (1, 2).

Although asymptomatic AF is detected in patients who are frequently symptomatic, it is common independently from permanent or paroxysmal type as first referral. In other words, the patient may have the first recurrent attack during the first diagnosis but also may develop permanent AF. This case is important to decide whether to start treatments to prevent AF-associated complications (1, 2).

\section{General Approach}

$\mathrm{AF}$ and atrial flutter (AFL) managements have similarities. The general approach to manage newly onset AF/AFL does not have significant changes. Either the rate control or rhythm control option is selected for stable patients. Rapid electric therapy is needed for hemodynamically unstable cases (4-8).

The factors that determine whether rate control or rhythm control strategies would be preferred are as follows: If the patient has a permanent AF, less symptoms, hypertension, aged $>65$ years, and has no history of congestive heart failure and antiarryhthmic drug failure, rate control is suggested. However, if paroxysmal or newly diagnosed AF exists, age is $<65$ years, and no hypertension but congestive heart failure aggravated with AF and no antiarryhthmic drug failure exists, rhythm control should be considered (4-8).

\section{Rate Control Strategy}

Beta-blockers (BB), non-dihydropyridine type calcium canal blockers (CCB), and digoxin and its combinations are recommended for heart rate control. For the patients with cardiac failure, $\mathrm{BB}+/-\mathrm{di}$ goxin and particularly BB as well as CCB (diltiazem, verapamil) or its combinations are preferred for the patients with coronary artery disease. If the patient has an advanced sedentary life style and if she/he does not have any comorbidity, digoxin may be preferred as a monotherapy $(1,2,4-8)$.

Although dronaderon was included in secondary line drugs for the rate control in the guideline of 2010, it is reported in the update report of 2012 that this drug should not be used in patients with permanent AF/AFL for the rate control (7). Use of dronedarone is indicated to convert recurrent paroxysmal and permanent $\mathrm{AF}$ into permanent sinus rhythm and as a short-term antiarryhthmic agent (4 weeks) after cardioversion. Combined use of dronedarone with digoxin or dabigatran is not recommended. It is also contraindicated in case of an unstable hemodynamic event and in grade 1-2 heart failure (2).

Physicians should control the heart rates of patients with AF to below 100 pulse/min before discharge from the emergency room.
The purpose of the long-term therapy is to control the heart rate in intermediate exercises such as the walking test to keep it below 110 pulse/min (1).

\section{Rhythm Control Strategy}

Rhythm control strategy is suggested for patients with AF/AFL who still present symptoms or who show a failure to control the rate by rate control therapy. The aim here is to recover symptoms and clinical outcomes and it never includes elimination of all the patients with $\operatorname{AF}(1,2,7,8)$. Although the conventional belief for long-term therapy is to prefer rhythm control, studies showed that there was no significant difference between rhythm control and rate control approaches in terms of clinical outcomes (4-6).

Three options are suggested for rhythm control: Rate decelerating agents, synchronized electrical or pharmacological cardioversion, and pre-treatment by antiarryhthmic drugs before electrical cardioversion. There are low-grade evidences that the last suggestion prevents recurrence and increases the achievement of cardioversion (4-7).

If electrical therapy would be chosen, defibrillator devices utilizing biphasic waveform should be preferred because of their high achievement rate obtained at the first shock. The initial energy level is recommended to be 150-200 joules (4-7).

If drug therapy would be preferred for rhythm control, dronedarone, flecainid, propophenole, or sotalol should be considered for patients with normal systolic function who do not have any history of congestive heart failure. Dronadorene should be used carefully in the patients who are on digoxin. Class I antiarrythmic drugs should be avoided for patients with coronary artery disease and should be combined with atrioventricular (AV) node blocker drugs. Amiadorone or catheter ablation should be considered for resistant cases. However, if there is a history of congestive heart failure or systolic dysfunction of the left ventricle, drugs should be preferred according to the ejection fraction (EF) value. If $\mathrm{EF}>35 \%$, amiadorone or sotalol may be preferred. Sotalol should be used with caution for patients whose EF is between $35 \%$ and $40 \%$ and who are under a risk for torsades de pointes (female, age $>65$ years, diuretic use). Amiadorone is reported as a single choice for $\mathrm{EF}<35 \%$. Catheter ablation is suggested for resistant cases (7).

Intravenous vernakalant, which is recently included in the guidelines for rhythm control, is recommended as an alternative to ibutilide in patients who have congenital heart disease and newly diagnosed AF. Intravenous vernakalant is also suggested for the chemical cardioversion of AF, which appears within the first 3 days after a cardiac surgery. The primary effect of vernakalant is on the atriums and it is a rapid-acting agent. Half of the patients return to sinus rhythm within $90 \mathrm{~min}$. Venakalant is contraindicated for the following conditions: hypotension (systolic blood pressure $<100 \mathrm{mmHg}$ ), acute coronary syndrome, grade 3-4 heart failure, severe aortic stenosis, and QT prolongation (2).

\section{Risk Assessment}

Use of predictive indexes is suggested to assess the risk for stroke and bleeding for all patients with AF (paroxysmal, recurrent, or permanent). The scoring systems of $\mathrm{CHADS}_{2}$ or $\mathrm{CHADS}_{2}$-VASC for the risk of stroke and HAS-BLED for the risk of bleeding are included in the guideline $(1,4)$. CHADS $_{2}$ scoring system includes the history of congestive heart failure, hypertension, age $>75$ years, diabetes 
mellitus, and stroke/transient ischemic attack (TIA)/thromboembolism. Stroke/TIA/thromboembolism is graded by 2 points, whereas all other items are graded by 1 point. $\mathrm{CHADS}_{2}$-VASC, in addition to the first scoring system, includes the parameters of vascular disease, age between 74 and 75 years, and female gender; 2 points are predicted for the age $>75$ years. Both scoring systems have similar and low predictive values. The CCS guideline suggests the use of $\mathrm{CHADS}_{2}$ because it has a simple and wider area of use $(1,4-7)$.

The importance of the use of indexes for the risk of bleeding is beneficial to ensure that risk factors are considered. The score is recommended to compare the relative risk of major bleeding against stroke. HAS-BLED index evaluates the parameters of hypertension, abnormal renal and hepatic functions, stroke, bleeding, unstable INR values, age ( $>65$ years), and drug and alcohol use. If the score is $\geq 3$, this means the patient needs a close follow-up. However, a score of $\geq 3$ does not mean that there is a contraindication for anticoagulant use $(1,4-7)$.

\section{Wolf Parkinson White Syndrome}

Intravenous procainamid and ibutilide may be preferred for patients with "Wolf Parkinson White Syndrome" if they are hemodynamically stable. There is an indication for urgent electrical cardioversion for hemodynamically unstable patients. AV node blockers (digoxin, $\mathrm{BB}, \mathrm{CCB}$, adenosine) are contraindicated for this patient group (4-6).

\section{Prevention of Thromboembolism}

The most significant complications related to AF are because of thromboembolism. The risk of thromboembolism depends on many underlying causes. The appearance of stillness and flow abnormalities in the left atrium; progressive atrial dilatation; endocardial wearing and endocardial abnormalities, including edematous infiltration of the extracellular matrix; blood component abnormalities, including hemostatic and platelet activation; and abnormalities of the growth hormone and inflammation are reported to cause thromboembolism (4).

There are two important factors in determining the treatment strategy for thromboembolism: Duration of AF and hemodynamic status of the patient. If the rhythm control strategy is selected and the AF/AFL duration of hemodynamically stable patient is $>48 \mathrm{~h}$ or unknown, an optimal rate control should be provided first. In this patient group, warfarin (INR between 2 and 3) or oral anticoagulant (OAC) treatment by dabigatran should be applied for 3 weeks before and for at least 4 weeks after cardioversion. If AF/AFL continues or repeats after cardioversion, anti-thrombotic treatment must be continued. If sinus rhythm is provided and maintained for 4 weeks after cardioversion, the risk of stroke should be determined according to the evaluation result (4-7).

If the duration of AF is known as $<48 \mathrm{~h}$ in a hemodynamically stable patient and rhythm control would be applied, an anticoagulant is not required unless the patient has high risk factors. However, if the patient has high risk factors (mechanical valve, rheumatologic heart disease, or recent history of stroke/TIA), OAC treatment is suggested for 3 weeks before and for at least 4 weeks after cardioversion (4-7).

Risk of stroke is based on when long-term OAC treatment is planned. OAC treatment planning is suggested for patients with a $\mathrm{CHADS}_{2}$ score $\geq 1$. Aspirin may be preferred for selected patients by considering the risk/benefit ratio for patients with a $\mathrm{CHADS}_{2}$ score of 1. For the low-risk group with a $\mathrm{CHADS}_{2}$ score of 0 , consideration of other risk factors is a new suggestion. Accordingly, OAC treatment is not recommended for patients without additional risk factors. Aspirin is suggested for the low-risk patient group with females or vascular diseases. For female patients with age $>65$ years or and vascular disease combination, OAC treatment is recommended despite a lower CHADS, score (7).

Thromboembolism prophylaxis is suggested by aspirin 75-325 $\mathrm{mg} /$ day and warfarin to maintain INR between 2 and 3 for patients with non-valvular AF. Although the INR value has self and interpersonal changes and severe food, drug and alcohol interaction, warfarin has some advantages. The advantages are long-term clinical experience, presence of a specific antidote, and existence of a specific test for the titration of anticoagulation (4). However, an update suggestion of the guidelines of 2012 is to prefer new OAC drugs such as dabigatran, rivaroxaban, and apixaban to warfarin if there is any indication for OAC. New OAC drugs act by binding to thrombin (dabigataran) and Factor Xa (rivaroxaban and apixaban) reversibly. While these drugs are as effective as warfarin in the prophylaxis of stroke, their ability to cause intracranial bleeding complications is significantly less than warfarin. However, the lack of an antidote to reverse the effect of these drugs and a specific test to show anticoagulant effects are important disadvantages. Dose reduction of dabigatran is possible for the age group of $>75$ years and is needed for the age group of $>80$ years (7). The indications of dabigatran according to the latest guidelines are as follows: stroke; history of TIA or systemic embolism; left ventricle ejection fraction below $40 \%$; presence of symptomatic heart failure; $>75$ years of age; $>65$ years of age; as well as accompanying DM, CAD, or HT history (2).

Renal function test is suggested at least once a year for all patients who are on OAC therapy. If the patient has an accompanying chronic kidney failure, she/he should be followed closely. The principals of OAC treatment for patients with an estimated glomerular filtration rate of $>30 \mathrm{~mL} / \mathrm{min}$ and $15-30 \mathrm{~mL} / \mathrm{min}$ and who do not undergo dialysis are the same as those with normal kidney functions. If treatment would be started, warfarin should be preferred. Neither OAC nor aspirin is recommended for patients with an estimated glomerular filtration rate of $<15 \mathrm{~mL} / \mathrm{min}$ because of the high risk of bleeding (7).

Antithrombic treatment is administered in urgent cardioversion cases; however, the clinical progress of the patient and duration of $\mathrm{AF} / \mathrm{AFL}$ are considered. If the duration of AF/AFL known for a hemodynamically stable patient is $>48 \mathrm{~h}$ or the duration is unknown, OAC by warfarin (INR between 2 and 3 ) or dabigatran should be administered for 3 weeks before and for 4 weeks after urgent cardioversion. If AF/AFL persists or repeats, antithrombic treatment should be continued. If return to sinus rhythm is achieved and maintained for 4 weeks, antithrombotic treatment is planned according to the risk of stroke (4-7).

If the known duration of AF/AFL is shorter than $48 \mathrm{~h}$, anticoagulation is not needed before or after cardioversion unless the patient has high risk-factors for stroke. However, if AF/AFL persists or repeats, antithrombotic treatment should be started. In case of high-risk factors, OAC treatment should be applied for 3 weeks before and at least for 4 weeks after urgent cardioversion (4-7).

If urgent cardioversion is planned for a patient who is hemodynamically unstable, antithrombotic treatment is planned according to duration of AF/AFL and whether high-risk factors exist. If the duration is shorter than $48 \mathrm{~h}$ and there is no high-risk factor, anticoag- 
ulation treatment is not applied. If the duration is shorter than $48 \mathrm{~h}$ but there are high-risk factors or if the duration is longer than $48 \mathrm{~h}$, intravascular unfractionated heparin or low-molecular weight heparin is administered before urgent cardioversion and OAC treatment is arranged for at least 4 weeks (4-7).

If experienced personnel and eligible conditions exist, transesophageal echocardiography is an alternative to 3-week anticoagulation before cardioversion. It is preferred for patients in whom early cardioversion is required and anticoagulation therapy is not accepted by the patient or patients with high-risk factors in terms of bleeding. Furthermore, such procedures should be considered for cases where the prevalence of thrombus in the left atrium and left atrial appendix is more likely $(1,2)$.

Intravascular unfractionated heparin or low-molecular weight heparin is administered before urgent cardioversion for patients who do not have any thrombus in transesophageal echocardiography and then OAC treatment is continued until target INR values are reached. If thrombus is detected, OAC treatment is applied for 3 weeks and transesophageal echocardiography is repeated. If no thrombus is detected after the control procedure, cardioversion is applied and OAC treatment for an entire lifetime is suggested. If thrombus exists again, the treatment strategy should be changed and the rate control strategies should be preferred $(1,2)$.

\section{Catheter Ablation}

Catheter ablation therapy should be considered for patients with symptomatic recurrent and paroxysmal AF and who are resistant to at least one antiarrythmic treatment. Furthermore, catheter ablation of the left atrium may be used as a first-line therapy for the selected patients with paroxysmal AF and who do not have any congenital heart disease $(1,2)$.

\section{Hospitalization}

Highly symptomatic patients with decompansated heart failure or myocardial ischemia accompanying AF and patients in whom sufficient rate control cannot be achieved should be hospitalized (4-6).

\section{Conclusion}

Prominent topics in the new AF guidelines include a new classification of AF, new OAC drugs, an approach on using scales to assess the risk of stroke and bleeding, and new suggestions on rhythm and rate control strategies. Following the updated data and the applica- tion of these innovative approaches by emergency medicine physicians during daily practice will create a difference.

Peer-review: Externally peer-reviewed.

Conflict of Interest: No conflict of interest was declared by the authors.

Financial Disclosure: The authors declared that this study has received no financial support.

\section{References}

1. European Heart Rhythm Association; European Association for Cardio-Thoracic Surgery, Camm AJ, Kirchhof P, Lip GY, Schotten U, Savelieva I, et al. Guidelines for the management of atrial fibrillation: the Task Force for the Management of Atrial Fibrillation of the European Society of Cardiology (ESC). Eur Heart J 2010; 31: 2369-429. [CrossRef]

2. Camm AJ, Lip GY, De Caterina R, Savelieva I, Atar D, Hohnloser SH, et al. 2012 focused update of the ESC Guidelines for the management of atrial fibrillation: an update of the 2010 ESC Guidelines for the management of atrial fibrillation. Developed with the special contribution of the European Heart Rhythm Association. Eur Heart J 2012; 33: 2719-47. [CrossRef]

3. Available from: http://www.ncbi.nlm.nih.gov/pubmed/?term=atrial+fibrillation+guidelines Erişim tarihi: 01.10.2013

4. Steill IG, Macle L; CCS Atrial Fibrillation Guidelines Committee. Canadian Cardiovascular Society atrial fibrillation guidelines 2010: management of recent onset atrial fibrillation and flutter in the emergency department. Can J Cardiol 2011; 27: 38-46. [CrossRef]

5. Gillis AM, Skanes AC; CCS Atrial Fibrillation Guidelines Committee. Canadian Cardiovascular Society atrial fibrillation guidelines 2010: implementing GRADE and achieving consensus. Can J Cardiol 2011; 27: 27-30. [CrossRef]

6. Gillis AM, Verma A, Talajic M, Nattel S, Dorian P; CCS Atrial Fibrillation Guidelines Committee. Canadian Cadiovascular Society atrial fibrillation guidelines 2010: rate and rhythm management. Can J Cardiol 2011; 27: 47-59. [CrossRef]

7. Skanes AC, Healey JS, Cairns JA, Dorian P, Gillis AM, McMurtry MS, et al. Focused 2012 Update of the Canadian Cardiovascular Society Atrial Fibrillation Guidelines: Recommendations for stroke prevention and rate/rhythm control. Can J Cardiol 2012; 28: 125-36. [CrossRef]

8. Fuster V, Ryden LE, Cannom DS, Crjins HJ, Curtis AB, Ellenbogen KA, et al. $2011 \mathrm{ACCF} / \mathrm{AHA} / \mathrm{HRS}$ focused updates incorporated into the ACC/ AHA ESC 2006 guidelines fort the management of patients with atrial fibrillation: a report of the American College of Cardiology Foundation/ American Heart Association Task Force on Practice Guidelines. Circulation 2011; 123: e269-e367. [CrossRef] 